\title{
Modelo Empresarial, Gestão de Inovação e Investimentos de Venture Capital em Empresas de Biotecnologia no Brasil
}

\author{
Valéria Maria Martins Judice \\ Adelaide Maria Coelho Baêta
}

\begin{abstract}
Resumo
Este artigo estuda exploratoriamente modelos empresariais, gestão de inovação e investimentos de venture capital em empresas de biotecnologia brasileiras. O trabalho empírico organiza-se por meio de entrevistas realizadas com diretores de P\&D numa amostra de 50 empresas selecionadas de uma população de 304, tendo sido orientado por duas questões principais: 1) Como se dá a gestão de inovação em tais empresas? 2) Com que freqüência elas buscam investidores venture? Os resultados obtidos indicam escassez de inovações e investimentos venture nas empresas pesquisadas. Possíveis explicações e conseqüências são apontadas, estruturando-se o artigo em 5 seções: a primeira define os termos e caracteriza biotecnologia; a segunda apresenta o referencial teórico, destacando características organizacionais de bioempreendimentos e suas relações com venture capital; a terceira descreve a metodologia do estudo empírico; a quarta discute os resultados obtidos e a quinta apresenta as reflexões finais e perspectivas de pesquisa futura.
\end{abstract}

Palavras-chave: biotecnologia; capital de risco; inovação; empreededor-cientista.

\begin{abstract}
Exploratory research of 50 biotechnology companies selected from a population of 304 has evidenced shortage of venture capital investment in Brazil. This paper presents theoretical background and context for analysis of Brazilian biotechnology corporate development, investigating possible explanation factors for this VC scarcity. The presentation is divided into five sections. The first section defines terms and introduces the background and context for analysis of biotechnology and bio-industry enterprise; the second section outlines the theoretical approach; the third describes the methodological procedures; the forth presents the results and discussion; the fifth part finalizes the paper with the concluding remarks and future perspectives for research.
\end{abstract}

Key words: biotechnology; venture capital; innovation; scientist-entrepreneur. 


\section{INTRODUÇÃO}

O termo biotecnologia refere-se a um conjunto de tecnologias habilitadoras (enabling technologies) que possibilitam utilizar, alterar e otimizar organismos vivos ou suas partes, células, organelas e moléculas, para gerar produtos, processos e serviços com aplicações econômicas em saúde humana e animal, agricultura e meio ambiente.

Por serem tecnologias aplicáveis em diversos setores e cadeias industriais, seria incorreto, a rigor, falar em biotecnologia como um setor ou uma indústria específica (OECD, 1999). A chamada bioindústria é a aplicação, em escala industrial e empresarial, dessas variadas tecnologias para a geração de produtos e serviços em diversos segmentos de mercado. Adotando a noção de cadeias produtivas, incorporando fornecedores de insumos e equipamentos e subsetores em proximidade tecnológica, este estudo abrange os segmentos de mercado apresentados no Quadro 1, abaixo.

\section{Quadro 1: Segmentos de Mercado em Biotecnologia}

1. Saúde Humana: diagnósticos, medicamentos, vacinas, utilização de biodiversidade.

2. Saúde Animal: veterinária (animais de grande porte e domésticos, pets), vacinas, probióticos, nutrição animal, aquacultura.

3. Agribusiness: genética de plantas, transgênicos, produtos florestais, ornamentais, medicinais, bioinsecticidas; biofertilizantes; inoculantes.

4. Meio ambiente : biorremediação, tratamento de resíduos, análises.

5. “Instrumental complementar”: software, internet, bioinformática, e-commerce, $\mathrm{P} \& \mathrm{D}$, consultorias.

6. Insumos industriais: química fina, enzimas, alimentos.

7. “Em sinergia” : biomateriais, biomedicina, nanobiotecnologia.

8. Fornecedores : equipamentos; insumos e matérias primas.

Fonte: Judice, 2001.

Trata-se de uma indústria emergente (classificação de Porter, 1986), que vem crescendo rapidamente nos últimos anos, tendo passado no período de 1993 a 1999, nos Estados Unidos, de US\$ 8 bilhões de receitas para um volume total de negócios da ordem de US\$ 47 bilhões (ERNST \& YOUNG, 2000). Estima-se 
que o número total de empresas bioindustriais no mundo se situe em torno de 4.000 a 5.000 empresas, com países e regiões de maior concentração, conforme o Quadro 2, a seguir.

Quadro 2: Número de Empresas de Biotecnologia - 2002

\begin{tabular}{|c|c|}
\hline Países/Regiões & N. de empresas \\
\hline Estados Unidos * & 1.457 \\
\hline União Européia * & 1.879 \\
\hline India ** & 500 \\
\hline Japão * & 400 \\
\hline Canadá $*$ & 416 \\
\hline Brasil *** & 304 \\
\hline Austrália ** & 200 \\
\hline México ** & 90 \\
\hline Argentina ** & 50 \\
\hline
\end{tabular}

Fontes:* Ernst \&Young, 2002, **BIO, 2002, *** Judice, 2001.

No Brasil, a bioindústria apresenta-se também em emergência e conta com uma população de 304 empresas, incluídas empresas multinacionais e fornecedoras de equipamentos e insumos (39\%). Mais de $80 \%$ delas estão concentradas nos Estados de São Paulo, Minas Gerais e Rio de Janeiro e estima-se que 60\% sejam micro e pequenas empresas, com até 50 postos de trabalho (JUDICE, 2001). Em termos de potencialidades nacionais, existem grandes expectativas de descoberta de medicamentos, alimentos e insumos industriais oriundos da biodiversidade brasileira amazônica, da mata atlântica e do cerrado. Entretanto, essas promessas ainda não se cumpriram e muito poucos empreendimentos nacionais se estabeleceram efetivamente na área.

A bioindústria gera produtos e serviços de alto valor agregado, empregos qualificados e, em geral, suas aplicações em saúde tendem a repercutir positivamente na melhoria da qualidade de vida das populações usuárias, embora outras aplicações sejam fonte de debate ético e preocupação ecológica. Muito se tem dito sobre o potencial da biotecnologia e sobre um futuro ciclo de inovações que dela poderá resultar. Até o presente momento, contudo, os mais importantes desenvolvimentos bioindustriais estão concentrados nos Estados Unidos e Europa (Reino Unido, Alemanha e França), tendo como principais elementos propulsores, de um lado, os investimentos de venture capital e sua habilidade de organização e conversão de empreendimentos científicos e acadêmicos em negócios cotados nas principais bolsas e mercados de ações e, de outro lado, grandes corporações das indústrias químicas e farmacêuticas que, mediante aquisições de pequenas empresas de biotecnologia, ampliam suas capacidades de inovação ou atuam na comercialização mais ampla dos produtos gerados. 
Não existem estudos ou avaliações em profundidade sobre como tais modelos de bioempreendendorismo internacionais se aplicam ao caso brasileiro. Tendo em vista a ausência de uma cultura de investimentos venture no Brasil, a imaturidade dos investimentos de altas tecnologias em mercados de capitais e a existência de pouquíssimos casos de aquisições de empresas de biotecnologia no Brasil $^{(1)}$, este artigo investiga como bioempreendedores brasileiros se estruturam, organizam e financiam seus processos de inovação e quais seriam a relevância e as perspectivas de os investimentos de venture capital efetivamente contribuírem para seu desenvolvimento empresarial.

\section{Referencial Teórico}

Três aspectos principais compõem a moldura teórica adotada neste estudo. O primeiro refere-se à organização industrial, aos atores e ao ambiente de negócios prevalecentes em biotecnologia e bioindústria. O segundo aborda as características de emergência industrial das atividades bioindustriais e o modo como se refletem na gestão de inovação nas empresas. Finalmente, o último aspecto aqui apontado é o das relações entre biotecnologia e capital de risco.

\section{Organização Industrial, Atores e Ambiente de Negócios em Biotecnologia}

Desde os primeiros desenvolvimentos realizados, nos anos 1970/80, quatro conjuntos de atores e um conjunto de infra-estruturas tecnológicas vêm sendo relevantes para a criação, produção e comercialização de inovações bioindustriais, a saber: 1) a pesquisa científica universitária; 2) as micro e pequenas empresas start ups, nascidas da pesquisa universitária ou de departamentos de P\&D de empresas (spin-offs); 3) as grandes empresas químicas e farmacêuticas atuantes no mercado de produtos das ciências da vida; 4) os investimentos de venture capital em suas modalidades (angel seed, mezzanino e equity); 5) as incubadoras de empresas e parques tecnológicos.

A pesquisa científica é a fonte básica de conhecimentos para o desenvolvimento de produtos e serviços biotecnológicos e gerou o modelo empresarial clássico do setor: o cientista-entrepresário e a típica micro e pequena empresa spin-off universitária. Esse modelo tem sido particularmente bem sucedido nos Estados Unidos, onde o fenômento emergiu (KENNEY, 1986; BULLOCK; DIBNER, 1995). Trajetórias similares têm sido estimuladas (ou emuladas) em outros países (ROBERTS; MIZOUCHI, 1989; LEX, 1995; 
JANK, 1995), consolidando como paradigma empresarial o cientista carismático e empreendedor.

As micro e pequenas empresas (MPEs) de biotecnologia proliferaram internacionalmente na última década, sendo elementos centrais no desenvolvimento estrutural da bioindústria. Por sua falta de habilidades gerenciais, dificuldades de financiamento, comercialização e marketing e, fundamentalmente, por sua incapacidade de lidar com o complexo regime regulatório e com os custos de propriedade intelectual (ROTHWELL; DODGSON, 1994), MPEs de biotecnologia, lideradas por empresários cientistas, tornaram-se participantes naturais de arranjos organizacionais especiais, tais como incubadoras e parques tecnológicos, geralmente de vinculação universitária. Tais ambientes ou infra-estruturas tecnológicas especiais ajudam a superar as dificuldades de empreendimento, aumentam a base de conhecimentos e o potencial de formação de redes, agrupamentos e clusters. Como ninhada de pássaros recém-nascidos, empresas de biotecnologia tendem a se manter juntas e em proximidade a grandes centros de pesquisa (CARR, 2003).

Grandes empresas químicas e farmacêuticas, por sua vez, sempre estiveram tradicionalmente vinculadas a universidades por sua base de conhecimentos. Com a emergência de MPEs de biotecnologia, criaram novos mecanismos de acesso a inovação, tais como transferência de tecnologia, licenciamento, participação acionária ou aquisição propriamente dita. A complementaridade de relações e vínculos entre os três conjuntos de atores (universidades, MPEs de biotecnologia e grande indústria química e farmacêutica) tem sido amplamente reconhecida (ARORA; GAMBARDELLA, 1990; PISANO, 1991), compreendida como o resultado do processo de amadurecimento e passagem por estágios cíclicos através dos quais a estrutura industrial de biotecnologia evolui (JOLY, 1999; BARBANTI; GAMBARDELLA; ORSENIGO, 1999).

Criadas nos Estados Unidos no final dos anos 1950, as empresas de investimento em pequenos negócios (SBICs) constituíram os primeiros fundos de venture capital $^{(2)}$ com aplicações em diversos segmentos, em especial em novos empreendimentos de alta tecnologia (FINGERL, 2001). A relevância deste tipo de investimentos resulta da necessidade de prover recursos financeiros de longo prazo e suporte a gestão de empresas emergentes - de grande potencial de crescimento, mas sem condições de buscar financiamento nas organizações tradicionais de crédito (EMRICH; BAETA, 2000).

Em 2001 registrava-se a existência de 365 fundos constituídos, com investimentos de US\$12,6 bilhões nos EUA (FINGERL, 2001). No Brasil, tais fundos são muito mais recentes, só tendo começado a se constituir no final de 1990 e 
início de 2000. No presente momento, operam no país entre 50 e 70 fundos de venture (PRADO, 2003; PAVANI, 2003), dos quais, três com algum tipo de orientação/inclinação para investimentos em biotecnologia, a saber: FIR Capital Partners, Votorantim Ventures e Rio Bravo.

\section{Gestão de Inovação em Biotecnologia}

Dois importantes elementos do processo de gestão de inovação em empresas intensivas em conhecimento são o ambiente de relações em que interagem os atores internos e externos e os mecanismos e coalizões que estabelecem para o desenvolvimento de novas tecnologias e mercados (BIGNETTI, 2002).

As inovações baseadas em conhecimento possuem o mais longo prazo de espera entre o aparecimento do novo conhecimento e sua aplicação e sua transformação em produtos e serviços para o mercado. Outra característica fundamental dessas inovações é que quase nunca resultam de um só fator, mas da convergência de vários tipos de conhecimento, nem todos científicos ou tecnológicos. Pela imprevisibilidade inata, seus riscos são altos (DRUCKER, 2002).

Tem sido dito que as empresas de biotecnologia atuam em modelo organizacional de arquitetura aberta (POWELL, 1999), na medida em que muitas de suas funções essenciais são providas a partir das coalizões externas realizadas. Assim, dificilmente uma só entidade será capaz de fornecer todo o conjunto de necessidades das empresas participantes do setor, envolvendo aspectos diversos, como pesquisa científica e tecnológica, testes clínicos, propriedade intelectual, financiamento, capitalização, manufatura, marketing e distribuição. Conseqüentemente, as parcerias, cooperações, alianças estratégicas e licenciamentos a grandes empresas químicas e farmacêuticas, joint ventures, participações acionárias, aquisições e incorporações, assim como investimentos seed, mezzanino e venture são todos elementos vitais ao avanço da bioindústria. Dificilmente, em seu atual estágio evolutivo, empresas de biotecnologia poderiam ser classificadas como estruturas permanentes ou fechadas; na verdade, trata-se antes de um campo organizacional em processo de emergência e construção social (POWELL, 1999)

A bioindústria se enquadra na categoria emergente na análise estrutural realizada por Porter (1986), que descreve o cenário de nascimento industrial como estágio tecnológico (e competitivo) de fluidez, incerteza e oportunidades.

Enquanto muitos subsegmentos industriais se consolidam e produzem resultados visíveis que ganham a confiança dos clientes e usuários (diagnósticos moleculares, medicamentos), outros experimentam estágios iniciais, enfrentando a resistência e confronto da opinião pública (transgênicos, clonagem para fins 
terapêuticos) ou, então, tropeçam em ausência ou excesso de regulamentação, longos prazos de maturação e validação tecnológica. As empresas são pequenas e fragmentadas e a organização da indústria é, em muitos sentidos, precária.

São características comuns à indústria emergente os processos de tentativa e erro, os comportamentos erráticos, já que predominam a incerteza tecnológica, a incerteza estratégica e os altos custos de produção. O surgimento de spin offs (empresas desmembradas de outras empresas ou de universidades e centros de P\&D) e start ups (empresas iniciantes) resulta da inexistência ou da presença de baixas barreiras à entrada, características da fase de emergência e da atratividade produzida pelos ganhos do pioneirismo no mercado. Os usuários e consumidores também são iniciantes e desconhecem os produtos/serviços, devendo nesse estágio ser informados sobre eles, até que possam ser capazes de prover feed back mais completo de suas necessidades e experimentos de consumo.

Uma série de barreiras estruturais e fatores limitantes constrangem a ação das empresas no estágio industrial emergente: a ausência de infra-estrutura de instalações, de canais adequados de distribuição e suprimento de serviços complementares necessários, a qualidade irregular dos produtos, as dificuldades de obtenção de matérias primas e componentes, a ausência de padronização, escala e externalidades de produção, além de um estado de confusão ou, às vezes, desconfiança por parte dos clientes e consumidores.

Em outra dimensão, há incertezas quanto à imagem e credibilidade das empresas iniciantes junto à comunidade financeira e, finalmente, há atrasos e transtornos na obtenção de aprovação das regulamentações que, pouco a pouco, se estabelecem (PORTER, 1986).

A todas essas características de incerteza, soma-se também um ciclo longo de maturação de produtos de bioindústria, o que resulta em investimentos e riscos altos, intensidade tecnológica e longo tempo em pesquisa, desenvolvimento, registro, manufatura e distribuição.

Do ponto de vista da inovação, as agendas de pesquisa e desenvolvimento de empresas de biotecnologia são estabelecidas por uma combinação de fatores de competência científica e foco, noção de acesso a mercados, senso de factibilidade da área de pesquisa, capacidade de networking, parcerias e cooperações, capacidade de romper barreiras culturais, persistência, tenacidade.

\section{Biotecnologia e Capital de Risco}

Tem sido observado que, à medida que as empresas start up, baseadas em 
conhecimento progridem em seu ciclo de vida, as aptidões gerenciais se tornam mais importantes do que as habilidades empresariais, como se os empreendedores atingissem um limite executivo, a partir do qual sua inabilidade gerencial se torna prejudicial à empresa (ZACHARAKIS; MEYER; DECASTRO, 1999).

Para Drucker (2002, p. 260), setores industriais, baseados no conhecimento, possuem momentos de ebulição e abertura de oportunidades de entrada ou janelas. O ciclo se alterna em momentos de fechamento, ou abalos, quando a indústria amadurece e se estabiliza, sendo o número de inovadores sobreviventes menor do que antes. Nessa situação

“... a não ser que a nova empresa se desenvolva como um novo negócio e assegure que esteja sendo "administrada”, ela não sobreviverá, não importa quão brilhante seja a idéia empreendedora, quanto dinheiro ela atraia, quão bons seus produtos, nem mesmo quão grande a demanda por eles".

Conforme visto, as empresas de biotecnologia necessitam, de maneira intensa, de informações e um conjunto de insumos ou inputs externos para complementação de suas capacidades tecnológicas, mesmo que as atividades de P\&D estejam internalizadas. Além da intensidade de relações entre empresas e universidades ou centros produtores de conhecimento, no caso de empresas de biotecnologia, outro importante componente de $\mathrm{P} \& \mathrm{D}$ é sua condução por empresários cientistas.

Por suas conexões acadêmicas e, principalmente, por sua percepção e profunda conscientização do papel do avanço científico e tecnológico em seu negócio, em estágios iniciais de sua empresa, o empresário cientista busca colaboradores que contribuam para o avanço da tecnologia, tendendo, em certo sentido, a supervalorizar o papel da pesquisa científica, o $\mathbf{P}$ do P\&D, em detrimento do desenvolvimento tecnológico e da colocação do produto no mercado. Os bioempreendedores têm sido freqüentemente flagrados em comportamentos empresariais impulsionados pela emoção da pesquisa, em atitudes visionárias, que parecem oscilar entre o sentido schumpeteriano de entrepreneur (SCHUMPETER, 1991) e a devoção carismática e missionária à ciência no sentido weberiano (WEBER, 1978). Conforme nota recente survey: "The businessmenscientists who are biotechnology entrepreneurs often seem driven by motives more complex than a mere desire to make money especially when they are trying to find treatments for disease" (CARR, 2003, p. 3).

Porém a empresa moderna não é baseada no líder schumpeteriano visionário ou na autoridade carismática ou missionária weberiana. Se na fase inicial de seu desenvolvimento existe pouca necessidade de sistemas de controle formais, porque 
os funcionários estão em constante comunicação face e face e é possível conduzir o negócio sem estruturas hierárquicas de subordinação (SIMONS, 1995), em estágios mais avançados, quando se inicia o processo de alavancagem financeira e capitalização, mediante a busca de associação com venture capital, a start up adquire maturidade e outros recursos empresariais e compreende melhor a contribuição de outras especialidades profissionais necessárias à consolidação de seu empreendimento.

Com o auxílio do investidor de risco, o empresário cientista ou o empreendimento intensivo em conhecimentos diversifica seus parceiros e colaboradores, introduzindo a administração profissional de executivos (CEOs), gerentes financeiros, comerciais e de marketing. Nesse movimento, atinge nível organizacional mais complexo, em que a produção industrial e a organização financeira do negócio se estabilizam em rotinas e trajetórias padronizadas, ou passam ao domínio da autoridade burocrática no sentido weberiano.

A rotinização burocrática dos negócios, introduzida pelo capitalista venture, é um momento crucial no desenvolvimento empresarial. A entrada do investidor de risco age como um freio aos imperativos ou à vontade apaixonada da pesquisa que dominam o fundador empreendedor cientista, formatando, reestruturando e racionalizando o plano de negócios e as tarefas administrativas e gerenciais que podem efetivamente conduzir às fases de crescimento, escala e consolidação da empresa. Se ao empreendedor cabem a gestão da cultura e visão empresariais, ao profissional administrador ou gerente cabe especialmente a gestão do desempenho (SADLER-SMITH; HAMPSON; CHASTON; BADGER, 2003).

De fato, tem sido evidenciado que empresas financiadas por investidores venture apresentam taxa mais alta de sucesso/sobrevida do que empresas não financiadas. Isso não significa que o investidor de risco saiba sempre escolher empresas vencedoras, já que cerca de $20 \%$ de empresas investidas em venture são zumbis (vivas-mortas), falhando em prover os retornos esperados pelo investidor (ZACHARAKIS; MEYER; DECASTRO, 1999). O ponto a ser enfatizado, portanto, não é a capacidade de antecipação do sucesso empresarial por parte do investidor venture, mas, sobretudo, sua capacidade de proativamente fomentar e garantir racionalmente o sucesso, particularmente por meio da gestão profissionalizada. Ao capitalista e investidor de risco cabe papel fundamental na solução do chamado dilema do fundador (ADIZES, 2002), a passagem da empresa da fase de adolescência para adulta.

De outro ângulo, nem sempre investidores venture se dispõem a aplicar em biotecnologia por terem aprendido, em sua experiência, que nem sempre os produtos de biotecnologia atingem seu potencial esperado de faturamento ou sustentação de margens de vendas e que muitas vezes, nem mesmo conseguem 
efetivamente chegar ao mercado: podem ocorrer falhas em testes clínicos, por exemplo. Um caminho de ganhos consistentes e contínuos em biotecnologia não é totalmente claro e esse é o aspecto mais importante na avaliação de investidores. Momentos de ceticismo e desinteresse por biotecnologia ocorrem por parte de investidores venture (CARR, 2003), diminuindo o acesso à capitalização de empresas. Afinal, após 30 anos de percurso, a bioindústria não pode usar a desculpa de sua juventude, tampouco é capaz de permanentemente recriar a aura de hipervalorização de seu imenso potencial futuro, como já vem há anos fazendo (ESPOSITO; OSTRO, 1998).

\section{Metodologia}

O material utilizado no estudo empírico constitui-se de entrevistas telefônicas realizadas com 50 diretores-sócios, gerentes, diretores de P\&D e executivos de empresas de biotecnologia em todo o país. As entrevistas foram realizadas com base em roteiro padrão, previamente elaborado, e foram transcritas e analisadas em seu conteúdo, tendo sido aproveitadas apenas aquelas, em número de 40 , que responderam completamente ao roteiro de questões utilizado.

Tendo em vista o caráter exploratório do estudo, não houve preocupação com rigorosa representatividade estatística na seleção, realizada de forma intencional. Os resultados observados e aqui reportados devem ser vistos, sobretudo, como indicadores de tendências gerais e como uma primeira incursão ao conhecimento das relações entre bioindústria brasileira e venture, esperandose que sejam orientadores de investigações mais aprofundadas em etapas posteriores.

É importante, entretanto, enfatizar que, por possuírem as autoras deste artigo experiência de muitos anos em pesquisa e trabalho profissional com empreendimentos bioindustriais, a investigação contou com a familiaridade e ampla compreensão do contexto, ambiente, principais desafios e dificuldades enfrentados pelos integrantes de tais empreendimentos.

Para os propósitos do presente artigo, as variáveis centrais investigadas foram as seguintes:

1. Internalização de $P \& D$

2. Existência de relações colaborativas com universidades 
3. Intensidade de P\&D e diversificação de equipe

4. Número de patentes concedidas e pleiteadas

5. Volume e valor de investimentos e financiamentos obtidos

No que se refere à caracterização da amostra, as Figuras 1 e 2 assinalam como ela se distribui relativamente à população, tendo em vista, respectivamente, a localização regional e a segmentação de mercado conforme classificação estabelecida pelo estudo. $\mathrm{O}$ intuito da apresentação destas informações é apontar que, embora intencional, sem estrito rigor estatístico e em caráter exploratório, o estudo empírico buscou e efetivamente logrou extrair uma amostra o mais semelhante possível à população identificada de empresas.

A Figura 1, abaixo, mostra a identidade amostra- população no que concerne à distribuição regional. Com exceção do Centro-Sul, que concentra a indústria, todas as demais regiões do país foram ligeiramente super-representadas.

\section{Figura 1: Distribuição Percentual de Empresas de Biotecnologia por Regiões Brasileiras População X Amostra ( N=304 e n=50)}

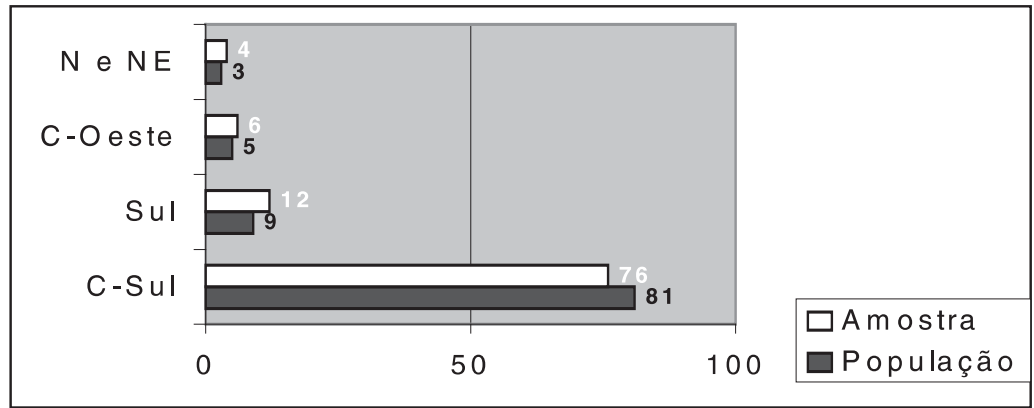

A Figura 2, por sua vez, mostra que para a distribuição dos segmentos de mercado, a situação da amostra é relativamente consistente com a população, exceto para segmentos que não obtiveram representatividade perfeita na amostragem. Optou-se intencionalmente por não priorizar empresas multinacionais (MNCs) e empresas fornecedoras de equipamentos e houve uma sub-representação não-intencional do segmento de Química Fina e Enzimas. 
Figura 2: Distribuição Percentual de Empresas de Biotecnologia por Segmentos de Mercado População x Amostra ( $N=304$ e n=50)

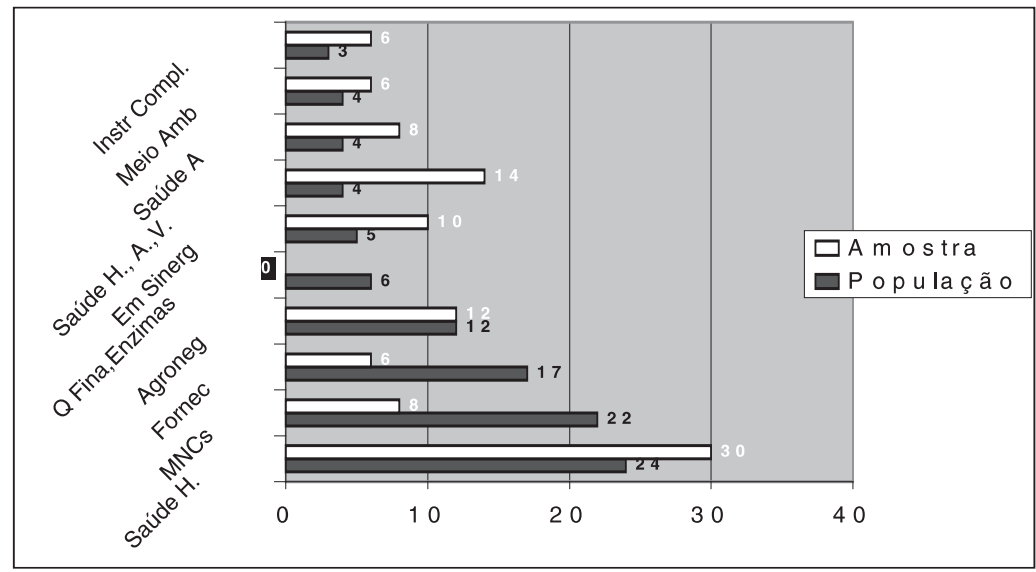

A ausência desse segmento, tipicamente de grande porte, ocorreu pelo fato de a amostra ter dado prioridade intencional a micro e pequenas empresas (MPEs) e a micro e pequenas empresas incubadas (MPEIs), que representam 85\% do total estudado. Em especial, a amostragem buscou dar margem mais ampla às MPEIs relativamente à sua proporção observada na população total, no sentido de obter mais conhecimento desse segmento mais necessitado de apoio e de políticas públicas específicas, além de buscar conhecer melhor como o instrumento incubação pode fortalecer a bioindústria nacional. As 15 empresas incubadas selecionadas na amostra se distribuíram da seguinte forma: 4 em São Paulo, 3 em Minas Gerais, 2 em Brasília, 2 no Rio Grande do Sul, 2 em Pernambuco, 1 no Rio de Janeiro, 1 em Santa Catarina. A Tabela 1, a seguir, mostra a distribuição da amostra em termos das dimensões empresariais.

Tabela 1: Dimensão das Empresas pelo Volume de Empregos Gerados

\begin{tabular}{|l|c|c|}
\hline \multirow{2}{*}{$\begin{array}{c}\text { Dimensão } \\
\text { (n. de empregados) }\end{array}$} & \multicolumn{2}{c|}{$\begin{array}{c}\text { Distribuição de Empresas } \\
(\mathbf{n = 4 0 )}\end{array}$} \\
\cline { 2 - 3 } & N.o & \% \\
\hline Micro - até 49 & 31 & 87 \\
\hline Pequena - de 50 a 100 & 3 & 15 \\
\hline Média/ Grande - mais de 100 & 6 & 100 \\
\hline Total & 40 & \\
\hline
\end{tabular}

Fonte: entrevistas realizadas. 


\section{Resultados e Discussão}

As informações coletadas foram sintetizadas e processadas, considerando aspectos quantitativos e qualitativos das relações e conteúdos apresentados na entrevista. A discussão dos resultados realiza-se a partir das tabelas montadas e sua análise combinada com resultados e informações obtidos a partir de outras pesquisas em temáticas assemelhadas e com o foco em biotecnologia e bioindústria.

\section{Gestão de Inovação na Bioindústria Brasileira}

Estudos empíricos recentes vêm indicando baixa capacidade inovadora de empresas da bioindústria brasileira (CASSIOLATO; LASTRES, 2000; FAJNZYLBER, 2001). Em amostragem com 18 empresas mineiras, Fajnzylber (2001) conclui pela existência de: a) baixa internalização de P\&D (30\% das empresas entrevistadas); b) alta utilização de conhecimento público ( $60 \%$ das empresas) e c) relativamente baixa taxa de inovação ( $40 \%$ das empresas com propriedade intelectual).

De outro ângulo, tais análises identificaram como pontos críticos ao desenvolvimento da bioindústria: a escassez de competências em gestão e conhecimento dos mercados; dificuldades de comercialização; ausência de estratégias de marketing; baixa visibilidade; falta de escala; altos custos de lançamento de novos produtos; e barreiras à entrada em mercados globais /internacionais (CASSIOLATO; LASTRES, 2000; FAZNZYLBER, 2001).

A existência internalizada de departamentos de P\&D tem sido considerada um componente essencial para a gestão de inovação, assimilação, adaptação e transformação de tecnologias e conhecimentos adquiridos externamente (GIBBONS; JOHNSTON, 1974; WALSH, 1993; FAULKNER; SENKER; VELHO, 1995; JOLY, 1999). Por outro lado, as necessidades de administração profissionalizada de empresas, baseadas em conhecimento, foi enfatizada no item 2.3 deste artigo.

Buscando avaliar como a bioindústria brasileira atua na gestão dos processos de inovação, equaciona o processo de crescimento e diversificação profissional de suas equipes e busca recursos para sua capitalização, o presente estudo avalia: 1) a internalização de $\mathrm{P} \& \mathrm{D} ; 2$ ) a capacidade de realizar cooperações tecnológicas com universidades e centros de pesquisa nacionais e internacionais; 3) intensidade de inovações indicada por número de patentes obtidas; 4) crescimento e profissionalização administrativa e qualificação da gestão versus dedicação a P\&D e 5) financiamentos e capitalização .

Os resultados encontrados parecem diferir substancialmente do padrão encontrado por Fajnzylber (2001), podendo ser visualizados na Tabela 2, que sugere 
estarem as empresas tecnologicamente abertas à contínua absorção de novos conhecimentos a serem utilizados na geração de novos produtos e serviços.

\section{Tabela 2: Internalização de P\&D e Ligações com Universidades e Centros de Pesquisa}

\begin{tabular}{|c|c|c|c|c|}
\hline \multirow{2}{*}{$\begin{array}{c}\text { Situação da } \\
\text { empresa }\end{array}$} & \multicolumn{2}{|c|}{$\begin{array}{c}\text { Empresas que possuem P\&D } \\
\text { internalizado } \\
(\mathbf{n}=\mathbf{4 0})\end{array}$} & $\begin{array}{c}\text { Empresas ligadas a universidades e } \\
\text { centros de pesquisa } \\
(\mathbf{n}=\mathbf{4 0})\end{array}$ \\
\cline { 2 - 5 } & $\mathbf{N . 0}$ & $\mathbf{\%}$ & $\mathbf{N . o}$ & $\%$ \\
\hline SIM & 36 & 90 & 37 & 93 \\
\hline NÃO & 4 & 10 & 3 & 7 \\
\hline TOTAL & $\mathbf{4 0}$ & $\mathbf{1 0 0}$ & $\mathbf{4 0}$ & $\mathbf{1 0 0}$ \\
\hline
\end{tabular}

Fonte: entrevistas realizadas.

Conforme se evidencia na Tabela 2, 90\% das empresas pesquisadas têm realizado desenvolvimento tecnológico próprio, possuindo departamentos dedicados a P\&D e 93\% possuem relações formais ou informais com universidades e centros de pesquisa, o que sugere que as empresas pesquisadas se apresentam adequadamente supridas de redes de apoio à pesquisa.

Embora esses achados difiram de outros resultados empíricos alcançados, parece importante identificar seus significados no que se refere à geração de inovação. Como indicador de intensidade de inovação, buscou-se, assim, conhecer o número total de patentes obtidas por empresas da amostra estudada. Diferentemente dos achados de Fajnzylber (2001), que não coloca seus resultados em contexto, isto é, não os compara a outros resultados obtidos, o número de patentes encontrado em nossa amostra parece ser expressivo, considerando-se os níveis de patenteamento nacional mais recentes.

O INPI, Instituto de Propriedade Industrial, teria registrado na área de Biotecnologia Farmacêutica 68 pedidos de patente em 1996; 150 em 1997 e 112 em 1998 (Arnt, 2001). No total as empresas pesquisadas apresentaram 47 patentes, com 21 dessas já concedidas e 26 solicitadas e em processo de obtenção. Considerando-se o conjunto estudado, a média por empresa é de quase uma patente, mas, na verdade, apenas 15 empresas ( $30 \%$ da amostra) de fato obtiveram ou buscaram patentes. Esses resultados finais colocam a amostra estudada em situação pior, em termos de inovação, relativamente à amostra pesquisada por Fajnzylber (2001), embora este pesquisador não tenha relativizado seus resultados, comparando-os a outros obtidos.

Lamentavelmente, as informações sobre patentes solicitadas deveriam ter sido mais detalhadas, em termos de sua distribuição ao longo do tempo, o que, entretanto, não ocorreu por falha no instrumento de coleta. Assim, as 47 patentes concedidas/solici- 
tadas encontradas reportam-se a toda a história das empresas e não somente ao momento e ano da pesquisa. Considerando-se que a idade média das empresas é de 10 anos, mesmo sem detalhadas comparações com outros setores, chega-se à conclusão que a atividade patentária das empresas não foi nada espetacular. Por outro lado, quando se considera que o ano marco para o registro de propriedade intelectual em biotecnologia no Brasil é 1996, ano de aprovação da legislação, esse desempenho fica relativamente melhor; entretanto, dada a falha da coleta, não se pode afirmar categoricamente o fato, mas apenas apontá-lo como possibilidade.

A verdade, porém, é que apenas uma empresa entre as 50 estudadas (40 informantes), com apenas 3 anos de existência, foi responsável pela obtenção e solicitação de 13 patentes, ou seja, quase um terço do total de patentes observadas no grupo, o que, de fato, é um resultado excepcional e merece ser estudado.

Analisando-se esse caso específico, além de excepcional capacidade de P\&D, que, como visto, a maioria possui, observam-se na empresa duas variáveis importantes, que poderiam justificar sua hiper-atividade patentária: 1) trata-se de empresa capitalizada, investida por capital de risco; e 2) trata-se de empresa eminentemente voltada para o mercado internacional, já certificada e buscando a inserção de seus produtos fora do país, particularmente nos Estados Unidos.

No que concerne à relação entre o ímpeto inovador e aquilo que no referencial teórico deste artigo se denominou "rotinização do carisma”, isto é, a expansão de administração profissional na empresa, a Tabela 3, a seguir, relaciona a intensidade de P\&D e tamanho e grau de maturidade da empresa, medidos pelo número de postos de trabalho.

\section{Tabela 3: Intensidade de P\&D, Surgimento de Outras Funções Profissionais e Crescimento}

\begin{tabular}{|c|c|c|c|c|c|c|}
\hline \multirow{2}{*}{\multicolumn{2}{|c|}{ N. de postos de trabalho }} & \multirow{2}{*}{\multicolumn{2}{|c|}{$\begin{array}{c}\text { Empresas } \\
(\mathbf{n}=39)\end{array}$}} & \multirow{2}{*}{$\begin{array}{c}\text { Intensidade em } \\
\text { P\&D } \\
\text { Tp\&d/Tpt(*) } \\
\%\end{array}$} & \multirow{2}{*}{\multicolumn{2}{|c|}{$\begin{array}{c}\text { Empresas } \\
(n=39)\end{array}$}} \\
\hline & & & & & & \\
\hline \multirow{3}{*}{ De 1 a 10} & \multirow{3}{*}{ Micro } & \multirow{3}{*}{17} & \multirow{3}{*}{44} & Até 50 & 5 & 13 \\
\hline & & & & & & \\
\hline & & & & $50-100$ & 12 & 31 \\
\hline \multirow{2}{*}{ De 11 a 49} & \multirow{2}{*}{ Micro } & \multirow{2}{*}{13} & \multirow{2}{*}{33} & Até 50 & 10 & 25 \\
\hline & & & & $50-100$ & 3 & 8 \\
\hline De 50 a 100 & Pequena & 3 & 8 & Até 10 & 3 & 8 \\
\hline \multirow[t]{2}{*}{ Mais de 100} & \multirow{2}{*}{$\begin{array}{l}\text { Média/ } \\
\text { Grande }\end{array}$} & \multirow[t]{2}{*}{6} & \multirow[t]{2}{*}{15} & $5-10$ & 5 & 13 \\
\hline & & & & 10 a 20 & 1 & 2 \\
\hline Total & & 39 & 100 & & 39 & 100 \\
\hline
\end{tabular}

Fonte: entrevistas realizadas (observar que $\mathrm{n}=39$ ).

(*)Total de pessoas em P\&D/Total de postos de trabalho. 
Chama a atenção neste caso, a micro empresa com até 49 postos de trabalho, que tende a ser altamente intensiva em P\&D, com de 50 a 100\% da equipe ocupando esta função e em casos mais extremos com ocupação de praticamente toda a equipe com P\&D. Cerca de $40 \%$ das empresas da amostra estudada têm de 50 a $100 \%$ de sua equipe integrando o departamento de P\&D: $31 \%$ das empresas com até 10 postos de trabalho e $8 \%$ das empresas com 11 a 49 postos de trabalho.

No outro extremo, estão empresas grandes que possuem 5 a $6 \%$ de sua equipe que, em valores absolutos, costuma ser muito mais do que os 10 funcionários da micro empresa, trabalhando em P\&D e, ao mesmo tempo, buscando todo tipo e variedade de fontes externas de conhecimentos, em relações formais e informais com universidades e centros de pesquisa nacionais e internacionais e possuindo administração profissionalizada.

\section{Financiamentos Convencionais e Investimentos Venture}

Em relação a financiamentos e investimentos, constatou-se uma necessidade generalizada de recursos, por parte de empresas de todos os portes, desde as micro e pequenas às grandes empresas. Todas precisam de recursos de forma a se equilibrarem em seu longo ciclo de maturação com elevados custos de desenvolvimento e produção, levando-se em conta um alto volume de importações na composição dos custos operacionais (de 50 a 100\%, conforme depoimentos).

Vinte e três das empresas estudadas, isto é, 58\% das declarantes são atualmente ou já foram, em algum momento de sua existência, financiadas. Um número total de 62 projetos de financiamento convencional foi identificado (2,7 projetos por empresa). Apenas 3 empresas (7,5\% das respostas válidas) ${ }^{(3)}$ foram capitalizadas por investidores venture, algumas com passagem e experiência em diversas etapas de capitalização (angel, seed e equity). Oito investimentos institucionais privados foram mencionados (20\% dos informantes válidos).

Registra-se que a questão de financiamento e capitalização foi percebida como o mais importante entrave ao crescimento do ponto de vista dos entrevistados. A maioria dos empresários convergiu em lembrar o fato de que fora do Brasil, particularmente nos Estados Unidos e Europa, empresas semelhantes às suas são mais bem nutridas e tratadas do ponto de vista de recursos financeiros em estágios iniciais do negócio e por claramente beneficiarem-se de políticas de apoio, fiscais e financeiras, por parte dos poderes públicos.

Empresários localizados fora de São Paulo lembraram o exemplo de tratamento dado a empresas de tecnologia pelos programas da FAPESP, no apoio à pequena empresa (PIPE - Programa de Inovação em Pequenas Empresas) e transferência 
de tecnologia universidade-empresas (PITE - Programa de Inovação Tecnológica Parceria Universidade-Empresas). Muitos sugeriram que esses modelos sejam adotados quer por agências financiadoras nacionais, quer por fundações estaduais de apoio à pesquisa. No caso destas últimas, merecem menção o caso da FACEPE, de Pernambuco, que já vem atuando no mesmo modelo FAPESP e, apoiando empresas de base tecnológica de Minas Gerais, a FAPEMIG, que vem desenvolvendo o Programa de Apoio a Micro e Pequenas Empresas de Base Tecnológica, PROMITEC, com características semelhantes aos programas PIPE e PITE da FAPESP, porém com muito menos recursos.

Embora sem informação neste estágio da pesquisa, acredita-se que outras fundações estaduais estejam também adotando o modelo paulista de financiamento a pequenas empresas de base tecnológica, tendo em vista seus resultados positivos e potenciadores de desenvolvimento regional.

\section{Reflexões Finais, Futura Pesquisa}

Buscando compreender exploratoriamente a relação entre modelos empresariais, gestão de inovação em biotecnologia no Brasil e perspectivas de investimentos de venture capital nesses segmentos industriais, as principais conclusões que emergem deste trabalho são as seguintes.

(1) Empresas de biotecnologia brasileiras apresentam comportamento paradoxal: se, de um lado, se dedicam fortemente à pesquisa e desenvolvimento, concentrando esforços e recursos humanos no respectivo departamento, de outro lado, possuem baixa intensidade de inovação, conforme indicado por obtenção de propriedade intelectual.

(2) É praticamente inexistente a cultura de investimentos venture em empresas de biotecnologia brasileiras, o que é parcialmente explicável pela ausência desse ator do cenário por um longo tempo. Apenas no final dos anos 1990 e início de 2000 surgiram os primeiros fundos com tal natureza.

(3) No modelo empresarial brasileiro de bioindústria, o empresário cientista tende a ser fortemente avesso a risco, ou avesso a venture. Constatou- se que é reduzido o percentual de empresas investidas (7,5\%), existindo uma clara preferência por financiamentos públicos (58\%); ou efetivamente nenhum financiamento: 35\% não acessam recursos financeiros externos.

Interpreta-se a preferência por financiamentos públicos como resquício ou importação da cultura prevalecente no local de origem dos bioempreendedores 
cientistas, isto é, o meio universitário. De outro lado, a inexistência de alternativa cultural venture, motivada pela ausência desse tipo de investimento no país até muito recentemente, resulta no desconhecimento e na resistência da bioindústria brasileira ao importante papel de tal ator na profissionalização gerencial, no impulso rumo à rotinização produtiva e comercial de empresas baseadas em conhecimentos, isto é, nascidas de empreendedores intelectuais e cientistas, sem formação ou gosto gerencial e motivados primordialmente pela excitação da pesquisa.

As próximas etapas de pesquisa centrarão esforços nos investidores venture, buscando compreender suas motivações e contribuições ao desenvolvimento da bioindústria e suas dificuldades relativamente à abordagem e captação de investimentos nesses empreendimentos.

\section{Artigo recebido em 09.10.2003. Aprovado em 28.05.2004.}

\section{Notas}

${ }^{1}$ O único caso de aquisição de que se tem conhecimento é o da BIOBRÁS, criada em 1976 (primeira empresa brasileira de biotecnologia) e adquirida em 2001 pela dinamarquesa Novo Nordisk, atual maior fabricante mundial de insulinas.

${ }^{2}$ Neste artigo, em lugar de capital de risco usamos a expressão em inglês venture, por julgá-la mais adequada à idéia original. A palavra venture é entendida como projeto ou atividade nova, excitante e difícil, envolvendo algum risco. A palavra risco, na língua portuguesa tem a conotação de atividade perigosa e não meramente aventureira (EMRICH; BAÊTA, 2000).

${ }^{3}$ Após a realização do estudo empírico, foram identificadas três novas empresas criadas por investidores de risco (Votorantim Ventures) em São Paulo, a saber: Allelyx, Scylla e CanaViallis, ligadas à genômica e bio-informática (maiores informações sobre essas empresas em BARELLI, S., Da incerteza do laboratório para o investimento de risco, Folha de São Paulo (Folha Sinapse), 24/ 09/2002: 22-24).

\section{ReferênCias Bibliográficas}

\section{ADIZES, I.}

Os ciclos de vida das organizações. como e por que as empresas crescem e morrem e o que fazer a respeito. $5^{\mathrm{a}}$ reimpressão de 1990, São Paulo: Thomson Pioneira, 2002.
ARNDT, R.

Tesouro verde - O Brasil aprende a transformar a fauna e a flora em riqueza. Exame, São Paulo, v. 9, n. 739, p. 52-64, 2 de maio 2001. 
ARORA, A.;

GAMBARDELLA,A.

Complementary and external linkages: the strategies of the large firms in biotechnology. The Journal of Industrial Economics, Essex, UK. V. 38, n.4, p. 361-379, june 1990.

\section{BARBANTI, P.;}

GAMBARDELLA, A.;

ORSENIGO, L.

The evolution of collaborative relationships among firms in biotechnology. International Journal of Biotechnology. Milton Keynes, UK, v. 1, n. 1, p. 10-29, 1999.

\section{BIGNETTI, L.P.}

O Processo de inovação em empresas intensivas em conhecimento. Revista de Administração Contemporânea, Curitiba, v. 6, n. 3, p. 33-53, set./dez. 2002.

BIO.

Biotechnology Industry Organization Annual Report. 2002. Disponível em: $<\mathrm{w}$ w w. b i o. o r g / l i n k s / international.asp>. Acesso em: 15 out. 2002.

BULLOCK, W. O.;

\section{DIBNER, M.D.}

The State of US biotechnology industry. Trends in Biotechnology, v. 13, n. 11, p. 463- 467, Nov. 1995. Disponível em: http:// www.sciencedirect.com/science>. Acesso em: 28 ouc. 2004.
CARR, G.

Climbing the helical staircase - A survey of biotechnology. The Economist, London, v. 366, n. 8316, p. 50, after: 1-20, 29 de mar. 2003.

CASSIOLATO, J.E.;

LASTRES, H. M. M.

Local systems of innovation in mercosur countries. Industry and Innovation, Copenhagen, Denmark. v. 7, n. 1, p. 33-54, june, 2000.

\section{DRUCKER, P.F.}

Inovação e espírito empreendedor: prática e princípios. $6^{\mathrm{a}}$ ed. São Paulo: Thompson /Pioneira, 2002.

EMRICH, G;

BAÊTA, A. M. C.

Capital de risco. In: FILION, L. J.; DOLABELA, F. (Colab.). Boa idéia! E agora? Plano de negócio, o caminho seguro para criar e gerenciar sua empresa. São Paulo: Cultura Editores Associados, p. 245-255, 2000.

\section{ERNST;YOUNG.}

The economic contributions of the biotechnology industry to the U.S. economy. Prepared for the Biotechnology Industry Organization by Ernst \& Young economics consulting and quantitative analysis. 2000. Disponível em: <http:// www.bio.org $>$. Acesso em: 15 dec. 2001.

Beyond borders - Ernst \& Young global biotechnology report. 2002. Global Biotechnology at a glance. Disponível em: <http://www.ey.com>. Acesso em: 20 oct. 2002. 
ESPOSITO, R.S.;

OSTRO, M.J.

Where have all the investors gone? The case for consolidation. Nature Biotecnology, London UK. v. 16, p. 63, Supplement: Bioentrepreneurship - Building a biotechnology company from the ground up. May. 1998. Disponível em: <http:// www.nature.com/cgitaf $>$. Acesso em: 28 oct. 2004

FAJNZYLBER, P.

Fatores de competitividade e barreiras ao crescimento no pólo de biotecnologia de Belo Horizonte. Belo Horizonte, CEDEPLAR/UFMG, 2001. 57 p. Texto para discussão.

\section{FAULKNER, W.;}

SENKER, J.;

VELHO,L.

Knowledge frontiers. public sector research and industrial innovation in biotechnology, engineering ceramics and parallel computing. Oxford, Claredon Press, 1995.

\section{FINGERL, E.R.}

Venture capital, inovação e a pequena empresa. Revista de Inteligência Empresarial, Rio de Janeiro, v. 1, n. 6, p. 37-43, Jan. 2001.

GIBBONS, M.;

JOHNSTON, R.

The roles of science in technological innovation. Research Policy, [S.L.] v. 3, n. 3, p. 220-242, nov. 1974. Disponível em: <http:// www.sciencedirect.com/science $>$. Acesso em: 28 oct. 2004.
JANK, B.

Biotechnology in european society. Trends in Biotechnology, [S.L.] v.13, n. 2 p.42-44, feb. 1995. Disponível em: <http://www.sciencedirect.com/ science >. Acesso em: 28 oct. 2004.

JOLY,P-B.

Introduction: innovations and networks in biotechnology. International Journal of Biotechnology, Milton Keynes, UK, n.1, p.1-9, 1999.

\section{JUDICE, V.M.M.}

Parque Nacional de Empresas de Biotecnologia. Estudo realizado para o Ministério de Ciência e Tecnologia, MCT. Relatório Final. 2001. Disponível em: <http:// www.mct.gov.br/Temas/biotec/ estudos_biotec_parque.htm>. Acesso em: 8 de out. 2003.

KENNEY,M.

Biotechnology: the universityindustrial complex. New Haven: Yale University Press, 1986.

\section{LEX, M.}

Promoting competitiveness of Biotechnology Industry. Trends in Biotechnology, [S. L.] v. 13, n. 2, p. 3941, feb. 1995. Disponível em: <http:// www.sciencedirect.com/science>. Acesso em: 28 oct. 2004.

\section{PAVANI, C.}

O capital de risco no Brasil conceito, evolução, perspectivas. Rio de Janeiro: E-Papers, 2003. 
PISANO, G.P.

The governance of innovation: vertical integration and collaborative arrangements in the biotechnology industry. Research Policy, [S. L.], v. 20, n. 3, p. 237-249, june 1991. Disponível em: <http://www.sciencedirect.com/ science $>$. Acesso em: 28 oct. 2004.

PORTER, M.E.

Estratégia competitiva: Técnicas para a análise de indústrias e da concorrência. $7^{\mathrm{a}}$ ed., Rio de Janeiro: Campus, 1986.

PRADO, $\mathrm{M}$.

Instituto propõe estímulo para negócios inovadores. Folha de São

Paulo, [S. L.], p. 1, B3, 21 de abr 2003.

POWELL, W.W.

The social construction of an organizational field: the case of Biotechnology. International Journal of Biotechnology, Milton Keynes, UK, n. 1, p. 42-66, 1999.

OECD.

Modern Biotechnology and the OECD. Policy Brief OECD. Paris. OECD, June 1999.

ROBERTS, E.B.;

MIZOUCHI, R.

Inter-firm technological collaboration: the case of japanese biotechnology. International Journal of Technology Management, Singapore, v. 4, n. 1, p. 43-61, 1989.

ROTHWELL, R.;

DODGSON, $\mathrm{M}$.

Innovation and size of the firm. In: DODGSON, M.; ROTHWELL, R. (Eds.). The handbook of industrial innovation. Paperback edition 1996.
Cheltenham: Edward Elgar, 1994.

Chapter 25, p. 310-324.

SADLER-SMITH, E.;

HAMPSON, Y. CHASTON;

BADGER, B.

Managerial behaviour, entrepreneurial style and small firm performance. Journal of Small Business Management, Morgantown WV, USA, v. 41, n. 1, p. 47-67, jan. 2003.

SCHUMPETER, J.A.

Essays on entrepreneurs, innovations, business cycles and the evolution of capitalism. Edited by Richard V. Clemence. New Brunswick and London: Transaction Publishers, 1991.

SIMONS, R.

Levels of control: how managers use innovative control systems to drive strategic renewal. Boston: Harvard Business School Press, 1995.

\section{WALSH, V.}

Demand, public markets and innovation in biotechnology. Science and Public Policy, New Brunswick, NJ, USA, v.20, p.138-156, june 1993.

\section{WEBER, $M$.}

Economy and society. Berkeley: University of California Press. 1978.

ZACHARAKIS, A.L.;

MEYER, GD.;

DECASTRO, J.

Differing perceptions of new venture failure: a matched exploratory study of venture capitalists and entrepreneurs. Journal of Small Business Management, Morgantown WV, USA, v. 37, n. 3, p. 1-14, july 1999. 